\title{
AN INTEGRABLE MODEL FOR HELENE
}

\author{
J.S. BEVILACQUA \\ Instituto de Matemática e Estatística, Universidade de São Paulo, Brasil \\ and \\ W. SESSIN \\ Instituto Tecnológico de Aeronáutica, Centro Técnico Aeroespacial, Brasil
}

\begin{abstract}
The goal of our work is to determine the influence of Enceladus in the motion of Helene. We constructed a model considering an oblated central body (Saturn) and three satellites (Enceladus, Dione and Helene) under the action of central forces. The development of the potential was made assuming small eccentricities, null inclinations and two resonances present in this system (2:1 between Enceladus and Dione and 1:1 between Dione and Helene). The mean Hamiltonian preserves terms derived of the oblateness of the central body and also terms of both resonant arguments. The auxiliary Hori's system generated by this Hamiltonian is completely integrable. In the solution for Helene we included the perturbative effects of Dione and Enceladus which extend the usual teatment.
\end{abstract}

Key words: Resonance - Oblateness - Hori's auxiliary system

\section{Hypothesis of the problem}

Considering an isolated four bodies system: Saturn, Enceladus, Dione and Helene with masses $M, m_{1}, m_{2}, m_{3}$ and the following hypotesis:

oblateness of Saturn $\left(J_{2}\right)$;

small eccentricities $\left(e_{i}\right)$;

null inclinations;

resonances:

2:1 between Enceladus and Dione,

1:1 between Dione and Helene;

only gravitational forces acts on the system;

coordinate system $(G ; x, y, z)$, where $G$ is the mass center of the system.

The potential was developed in function of this coordinate system $(G ; x, y, z)$ using the classical theory (Brouwer and Clemence (1961)) except for the term involving the mutual atraction of Dione and Helene, when the ratio $\left(a_{1} / a_{2}\right)$ approaches to zero. For this case see Hori (1985). 


\section{Equations of motion}

Introducing Delaunay's variables the canonical equations of motion are:

$$
\frac{d\left(X_{i}, Y_{i}\right)}{d t}=\frac{\partial F}{\partial\left(\lambda_{i}, \varpi_{i}\right)}, \quad \frac{d\left(\lambda_{i}, \varpi_{i}\right)}{d t}=-\frac{\partial F}{\partial\left(X_{i}, Y_{i}\right)} \quad i=1,2,3
$$

and $F$ is the Hamiltonian given by

$$
F=F_{0}+R
$$

with

$$
\begin{gathered}
F_{0}=\sum_{i=1}^{3} \frac{\mu^{2} m_{i}^{3}}{2 X_{i}{ }^{2}}, \\
R=F_{1 / 3}+F_{2 / 3}+F_{1} .
\end{gathered}
$$

$F_{0}$ generates Keplerian motion of Enceladus, Dione and Helene with focus $G$; and the disturbing function $R$ is developed in terms ofDelaunay's elements. Taking $\left(\frac{m_{s}}{M}\right)$ as the small parameter $\varepsilon$ and assuming that $J_{2}$ and $e_{i}{ }^{2}$ (square eccentricities) are of order $\varepsilon^{\frac{1}{3}}$ and $J_{4},\left(\frac{m_{1}}{M}\right),\left(\frac{m_{2}}{M}\right)$ of order $\varepsilon^{\frac{2}{3}}$, the expressions in $R$ were developed up to $\varepsilon$ and rearranged.

\section{Intermediary orbit}

The short period terms were eliminated by Hori's method considering the existence of the resonances 2:1 and 1:1. Substituting in the Hamiltonian a new set of canonical variables:

$$
\begin{gathered}
x_{1}=X_{1}^{*}+\frac{X_{2}^{*}}{2}+\frac{X_{3}^{*}}{2}, \quad x_{2}=-\left(\frac{X_{2}^{*}}{2}+\frac{X_{3}^{*}}{2}\right), \quad x_{3}=\frac{X_{3}^{*}}{2}, \quad y_{i}=Y_{i}^{*}, \\
\lambda_{i}=\lambda_{i}^{*}, \quad \theta_{2}=\lambda_{1}^{*}-2 \lambda_{2}^{*}, \quad \theta_{3}=2\left(\lambda_{3}^{*}-\lambda_{2}^{*}\right), \quad \varpi_{i}=\varpi_{i}^{*}, \quad i=1,2,3
\end{gathered}
$$

Since $\lambda_{1}$ is a ciclic variable, $x_{1}$ is a first integral. The study of the resonant terms will be done in the neighbourhood of the exact resonances:

$$
\begin{gathered}
\left.\frac{\partial F_{0}^{*}}{\partial x_{2}}\right|_{x_{2}=0}=-\frac{\mu^{2} m_{1}^{3}}{\left(x_{1}+x_{2}\right)^{3}}-\frac{\mu^{2} m_{2}^{3}}{4\left(x_{2}+x_{3}\right)^{3}} \\
\left.\frac{\partial F_{0}^{*}}{\partial x_{3}}\right|_{x_{3}=0}=-\frac{\mu^{2} m_{2}^{3}}{4\left(x_{2}+x_{3}\right)^{3}}-\frac{\mu^{2} m_{3}^{3}}{4 x_{3}^{3}}
\end{gathered}
$$


and the Hamiltonian after these developments is given by

$$
F^{* *}=F_{2 / 3}^{* *}+F_{1}^{* *}
$$

and the auxiliary equations will be generated by $F_{2 / 3}^{* *}$ :

$$
\begin{gathered}
F_{2 / 3}^{* *}=\left(z_{2}^{* *}\right)^{2}+\beta_{1} e_{10} \cos \left(\theta_{2}^{* *}+\varpi_{1}^{* *}\right)+\beta_{2} e_{20} \cos \left(\theta_{2}^{* *}+\varpi_{2}^{* *}\right) \\
+\beta_{0}\left(z_{3}^{* *}\right)^{2}+\beta_{3} \cos \left(\theta_{3}^{* *}\right)+\beta_{4} \operatorname{cossec}\left(\theta_{3}^{* *} / 2\right)
\end{gathered}
$$

where

$$
z_{2}^{* *}=\frac{x_{2}}{x_{20}}-q_{2} \quad z_{3}^{* *}=\frac{x_{3}}{x_{30}}-q_{3}
$$

with $q_{2}, q_{3}, \beta_{k}, k=0,1,2,3,4$ are constants.

The auxiliary equations are :

$$
\begin{aligned}
& \frac{d z_{3}^{*}}{d \Lambda}=-\beta_{1} e_{10} \sin \left(\theta_{2}^{* *}+\varpi_{1}^{* *}\right)-\beta_{2} e_{20} \sin \left(\theta_{2}^{* *}+\varpi_{2}^{* *}\right) \\
& \frac{d y_{i}^{* *}}{d \Lambda}=-\beta_{1} e_{10} \sin \left(\theta_{2}^{* *}+\varpi_{1}^{* *}\right) \\
& \frac{d y \ddot{~}}{d \Lambda}=-\beta_{2} e_{20} \sin \left(\theta_{2}^{* *}+\varpi_{2}^{* *}\right) \\
& \frac{d z_{3}^{*}}{d \Lambda}=-\beta_{3} \sin \left(\theta_{3}^{* *}\right)-\frac{\beta_{4}}{2} \frac{\cos \left(\theta_{3}^{*} / 2\right)}{\sin ^{2}\left(\theta_{3}^{* *} / 2\right)} \\
& \frac{d y y_{3}^{*}}{d \Lambda}=0 \\
& \frac{d \theta_{2}^{*}}{d \Lambda}=-2 z_{2}^{* *} \\
& \frac{d \varpi_{i}^{* *}}{d \Lambda}=\frac{c_{1} \beta_{1}}{e_{10}} \cos \left(\theta_{2}^{* *}+\varpi_{1}^{* *}\right) \\
& \frac{d \varpi_{3}^{* *}}{d \Lambda}=\frac{c_{2} \beta_{2}}{2 e_{20}} \cos \left(\theta_{2}^{* *}+\varpi_{2}^{* *}\right) \\
& \frac{d \theta_{3}^{* *}}{d \Lambda}=-2 \beta_{0} z_{3}^{* *} \\
& \frac{d \varpi_{3}^{* *}}{d \Lambda}=\frac{3 P_{03}}{2 e_{30}}
\end{aligned}
$$

and this system has two first integrals

$$
\begin{gathered}
E=F_{2 / 3}^{* *} \\
G=\frac{d z_{2}^{* *}}{d \Lambda}-\frac{d y_{1}^{* *}}{d \Lambda}-\frac{d y_{2}^{* *}}{d \Lambda}
\end{gathered}
$$

Except by the equations in $y_{3}^{* *}$ and $\varpi_{3}^{* *}$ which are immediately integrated, the equations in $z_{3}^{* *}$ and $\theta_{3}^{* *}$ involve the solution of a general ideal resonant problem:

$$
\begin{gathered}
E=\beta_{0}\left(z_{3}^{* *}\right)^{2}+\beta_{3} \cos \left(\theta_{3}^{* *}\right)+\beta_{4} \operatorname{cossec}\left(\theta_{3}^{* *} / 2\right) \\
4 \beta_{0}\left(\Lambda-\Lambda_{0}\right)=\int \frac{d \theta_{3}^{* *}}{\sqrt{E-\beta_{3} \cos \left(\theta_{3}^{* *}\right)-\beta_{4} \operatorname{cossec}\left(\theta_{3}^{* *} / 2\right)}}
\end{gathered}
$$


The other equations are identical to the auxiliary system of Salgado and Sessin (1985), so the solution is obtained in terms of the Sessin's variables

$$
\begin{array}{cc}
z=z_{2}^{* *} & \theta=\theta_{2}^{* *} \\
H=\frac{D_{1}}{c_{1}} h_{1}-2 D_{2} h_{2} & K=\frac{D_{1}}{c_{1}} k_{1}-2 D_{2} k_{2}
\end{array}
$$

where $D_{i}, c_{i}, i=1,2$ are constants and

$$
h_{i}=e_{i 0} \sin \left(\theta_{2}^{* *}+\varpi_{i}^{* *}\right) \quad k_{i}=e_{i 0} \cos \left(\theta_{2}^{* *}+\varpi_{i}^{* *}\right), \quad i=1,2 .
$$

\section{Concluding remarks}

Substituting initial conditions (Voyager's data for Helene and Nautical Almanac for Enceladus and Dione) we obtained $30^{\circ}$ for the amplitude of Helene's libration with $\theta_{3}^{* *}$ centered at $59.96^{\circ}$, which is very close to the observational data. However, the same do not occur with the period of Helene's libration.

\section{References}

Brouwer, D., Clemence, G.M.: 1961, Methods of Celestial Mechanics, (New York: Academic Press). Hori, G-I.: 1966, Publ.Astron.Soc.Japan 18,287;

Salgado, T.V., Sessin, W.:1985 in Resonances in the Motion of Planets, Satellites and Asteroids, (São Paulo: Universidade de São Paulo), 93.

Sessin, W., Ferraz-Mello, S.:1984, Celestial Mechanics 32, 307. 